Cite this: Phys. Chem. Chem. Phys. 2014, 16, 792

Received 22nd September 2013, Accepted 29th October 2013

DOI: $10.1039 / c 3 c p 54011 a$

www.rsc.org/pccp

\section{Contribution of Coulomb explosion to form factors and mosaicity spread in single particle $X$-ray scattering}

\author{
Andrea Debnarova, ${ }^{a}$ Simone Techert*ab and Stefan Schmatz*c \\ The Coulomb explosion of the octamer water cluster has been studied employing time-dependent \\ density functional theory explicitly accounting for the laser field and thus not imposing any constraint on \\ the interaction between the laser pulse and the cluster. We focus on the effects of electron density \\ changes in the system under high-intensity $\left(10^{16}\right.$ and $\left.10^{15} \mathrm{~W} \mathrm{~cm}^{-2}\right)$ soft $X$-ray laser pulses and their \\ fingerprint in the reciprocal space, namely the ultrafast changes in $X$-ray diffuse scattering signals in \\ $k$-space (in the investigated $k$-space range from $10^{-3}$ up to $10 \AA^{-1}$ ). The present simulations indicate \\ that diffusional components in $X$-ray intensity changes propagate from low reciprocal resolution \\ (resembling the small-angle $\mathrm{X}$-ray scattering regime) to very high resolution (the wide-angle $\mathrm{X}$-ray \\ scattering regime) during the Coulomb explosion process.
}

\section{Introduction}

Among the various possible applications of free electron laser (FEL) science a major task is real-time monitoring of chemical reactions in the bulk liquid phase employing soft X-ray FEL radiation. XUV radiation classically allows core-shell excitation of light elements like nitrogen, carbon or oxygen which are the atoms biochemical systems (sugars, amino acids, proteins, DNA etc.) consist of. Similar to FEL imaging techniques, radiation damage is one of the limiting factors in FEL spectroscopy where it is postulated that radiation damage sets a limit for the probe-pulse intensity a sample can be exposed to. At high laser intensities, the energy transfer from the X-ray field to the molecules or clusters in the sample becomes dominant. Due to single- and multi-photon excitations the sample is transformed on a very short time-scale into a collection of highly charged ions embedded in a quasi-free electron gas. This process, the Coulomb explosion, which influences any measurement performed on the sample in several ways is generally divided into three main time-phases: in the first phase, which is dominated by photoionizations, the laser light couples to the atoms as if they were isolated, while the cluster environment does not have any effect. In the second phase the

\footnotetext{
${ }^{a}$ Max Planck Institute for Biophysical Chemistry, IRG Structural Dynamics of (bio)chemical Systems, Am Fassberg 11, 37077 Göttingen, Germany. E-mail: adebnar@gwdg.de, stecher@gwdg.de

${ }^{b}$ Deutsches Elektronensynchrotron DESY, Notkestraße 85, 22607 Hamburg, Germany

${ }^{c}$ Institut für Physikalische Chemie, Universität Göttingen, 37077 Göttingen, Germany.E-mail: sschmat@gwdg.de
}

cluster expands due to the strong electrostatic repulsion forces between the ions created in the first phase, together with 'inner ionized' or 'quasi free' electrons, highly energetic particles still bound to the cluster. Finally, the third phase is characterized by energy redistribution and recombination processes. ${ }^{1,2}$

For soft X-ray FEL sources the current intensity levels are close to the Coulomb explosion limits. It is theoretically straightforward to avoid the problem with the sample disintegrating during spectroscopic measurements: according to the more generalized FEL principle of collect and destroy, the probe pulse must be both intense and short enough to provide spectroscopic information before structural and electronic changes due to the Coulomb explosion erode the collected spectroscopic data. An important question concerning structural measurements employing strong X-ray laser pulses is how the behavior of the Coulomb explosion changes with the field strength. As the pulse lengths generally considered are in the order of 10 to 100 femtoseconds, the structural changes during this time have to be small if a strong and well-defined scattering signal is to be obtained.

Time-dependent density functional theory (TDDFT) in its formulation beyond linear response provides a promising theoretical tool for the detailed description of laser-matter interaction. TDDFT is a convenient method for providing a detailed description of the electron-gas which has a dominant effect on the structural data deterioration. The dynamics of molecules under the influence of fields with very high intensities and very short pulse lengths, as provided by an FEL, is highly non-linear. Full TDDFT offers a quantum-chemical approach to the problem contrary to the widely used molecular 
dynamics treatment. ${ }^{9-13}$ The coupling between the laser field and the molecular system is formulated in a semi-classical way, with the laser treated as a classical electromagnetic field.

The theoretical treatment of the Coulomb explosion problem incorporates a number of approximations. To simplify the problem it is assumed that the laser couples to the electrons only, whereas the coupling to the atomic nuclei can be neglected, an approximation used in the type of calculations where the laser-matter interaction is accounted for stochastically. Through the explicit presence of the laser field in the Hamiltonian, TDDFT beyond linear response, however, accounts even for the small effect the strong laser field exerts on the nuclei. The TD density functionals for the description of electrons in strong fields are still in the developmental stage. Therefore, experiments such as time-dependent scattering measurements are invaluable for gaining further information concerning the behavior of electronic structures in strong fields and will help to improve the precision of the computational methods and the quality of TD density functionals.

However, the size of the studied system is limited because this quantum-chemical approach is computationally expensive. Thus, the octamer $\left(\mathrm{H}_{2} \mathrm{O}\right)_{8}$ water cluster with $S_{4}$ symmetry was chosen as a test system (see Fig. 1) in our calculations, ${ }^{3}$ being small enough to keep the computational costs of TDDFT

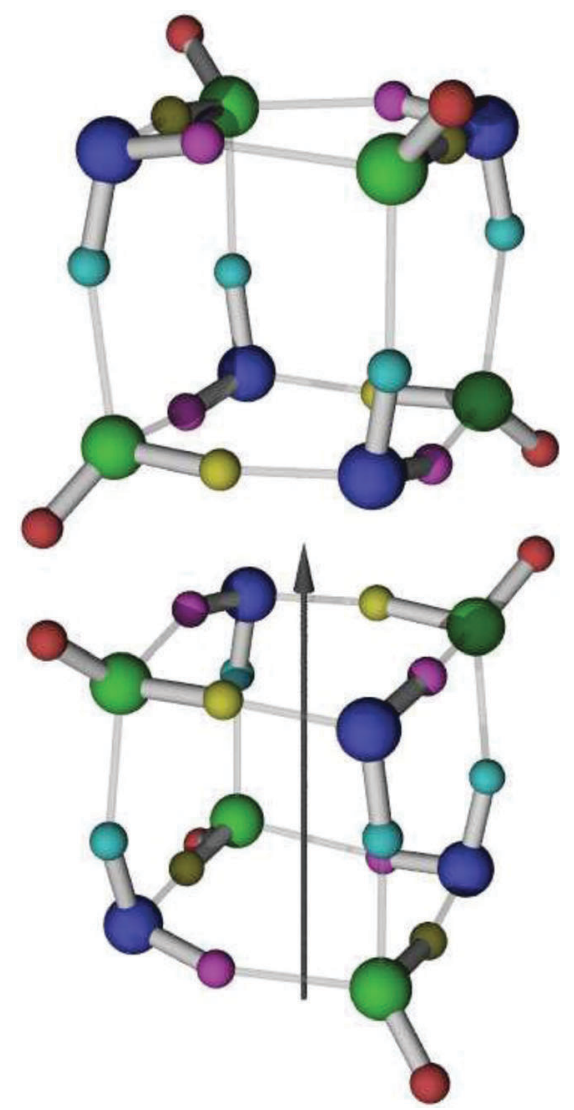

Fig. 1 Octamer water cluster $\left(\mathrm{H}_{2} \mathrm{O}\right)_{8}$ with symmetrically independent atom groups colored separately. The arrow indicates the axis of the $C_{4}$ rotation, which followed by a reflection with respect to the horizontal plane is the generator of the $S_{4}$ symmetry group of the cluster. reasonable, yet allowing for the inclusion of volume effects, e.g. free electron-gas shielding. The water octamer, characterized by its tri-coordinated hydrogen-bond deficient water structure, has been an object of both experimental ${ }^{4}$ and theoretical ${ }^{5}$ studies. The geometries of the two energetically lowest water octamer conformations are described by cubes where the eight water molecules which define the corners are oriented to form $D_{2 \mathrm{~d}}$ or $S_{4}$ symmetric structures. Opposed to the tetra-coordinated water that dominates the bulk, the tri-coordinated structures form water ${ }^{6}$ and ice ${ }^{7}$ surfaces.

The aim of the current study is to provide, via electron-density changes, further insight into soft X-ray FEL-matter interaction in a system which is relevant for chemistry under real conditions. Here, we focus in particular on electron density changes enforced by Coulomb explosions and their influence on possible $\mathrm{X}$-ray scattering patterns. In a previous study ${ }^{3}$ we examined the changes in total energy and kinetic energy during the Coulomb explosion process. The oxygen ions move relatively slowly even in the strongest field preserving the overall structure of the cluster. ${ }^{3}$

In the following, we study the Coulomb explosion behavior in a field of $10^{16} \mathrm{~W} \mathrm{~cm}^{-2}$ intensity for the soft X-ray regime of $120 \mathrm{eV}$. We study at a time interval of 5 femtoseconds and, for comparison, we consider the weaker field with an intensity of $10^{15} \mathrm{~W} \mathrm{~cm}^{-2}$ as well. Contrary to Dixit and Santra ${ }^{8}$ who focused on wavepacket dynamics in helium we concentrate on a much larger system, the octamer water cluster.

\section{Computational details}

In the framework of TDDFT, the TD Kohn-Sham equations must be solved explicitly to treat the interaction between matter and strong laser fields, an approach which is implemented in the program package OCTOPUS ${ }^{14,15}$ according to the description in our earlier publication. ${ }^{3}$ The total molecular system consists of two coupled dynamical systems, (a) an ensemble of non-interacting electrons (treated by the Kohn-Sham equations) and (b) the classically described atomic ions. The external electromagnetic laser field is also described classically within the dipole approximation as an additional external potential:

$$
E_{\text {laser }}(\mathbf{r}, t)=E_{0} f(t) \sin (\omega t) \hat{\mathbf{p}} \cdot \mathbf{r}
$$

with field strength $E_{0}$, frequency $\omega$, the envelope of the pulse $f(t)$ and the polarization vector $\hat{\mathbf{p}}$. This is always justified for optical wavelengths, while in the case of hard X-rays it is satisfied in the case of inner-shell electrons only.

As the OCTOPUS program employs pseudo potentials for the description of the core electrons, the laser wavelengths have to be larger than the oxygen $1 \mathrm{~s}$ absorption edge located at $4 \mathrm{~nm}$. We chose the soft X-ray wavelength of $10 \mathrm{~nm}$, corresponding to a photon energy of $120 \mathrm{eV}$, which can already be obtained with the FLASH-FEL at the DESY in Hamburg. Thus, in principle all 64 valence electrons of the octamer water cluster treated in this work can be excited in the simulation the time step of which is chosen to be 0.1 attoseconds. With respect to the size of the cluster, the description is kept well within the dipole 
approximation. Interaction between core electrons is not accounted for explicitly.

In our calculations, we make use of the ALDA approach suffering from well-known shortcomings such as self-interaction effects, while having the advantage of being a simple, extensively studied model. The perturbative treatment in this approximation ${ }^{16}$ causes problems of linear response TDDFT in describing charge-transfer excitations which however are not expected to appear in the present calculation, which goes beyond perturbation theory by employing explicit time-integration.

There are important differences between hard X-ray and soft X-ray laser-matter interactions. The higher energy end of the water absorption spectrum is dominated by the two main lines: the L-edge at about $30 \mathrm{eV}$ and the K-edge at about $530 \mathrm{eV} \cdot{ }^{17,18}$ The main contribution to electron scattering events employing hard X-ray photon pulses comes from Auger electrons ${ }^{19}$ because of the very small inelastic cross section for scattering events with high-energy photoelectrons which are expected to leave the sample very fast. In the case of soft X-ray pulses, the photoelectrons have energies in the range where the electron scattering cross section is high and thus contribute to the 'inner ionized' electron gas-formation. For strong fields these cross sections have to be modified to take multi-photon effects into account. These are included in the present calculation because the field is explicitly accounted for in the simulation (in contrast to other approaches based on perturbation theory).

The particular interest of the present work lies in the quantum-chemical description of electron density changes during the very initial processes that are induced by FEL pulses onto the octamer water cluster. The FEL pulse width of 7 fs consists of time-displaced 500 attosecond pulses in order to oversample the dynamic electronic processes induced by the pulsed FEL photons. The rising time of the whole FEL pulses is simulated by very narrow Gaussian functions. Since the electronic decay times (Auger, photoelectrons etc.) are on the order of some femtoseconds, these relaxation processes are slower than the incoming FEL pulse photons leading to an accumulation of kinetic energy.
The calculations start at 1 FWHM left of the pulse maximum, a reasonable choice according to comprehensive test calculations performed. ${ }^{3}$ Since the building-up phase is not relevant for the final results, our figures show data counted from the pulse maximum so that data corresponding to 'negative' times are not shown. Moreover, only a time interval of $5 \mathrm{fs}$ is considered while calculations have been carried out up to $200 \mathrm{fs}$, which proved to be sufficient since after 10 fs obviously no movement of the nuclei was recorded. The production of Auger electrons takes place within the first $6 \mathrm{fs}^{20}$

The sizes of the cubic spatial boxes (spatial boxes of different size have been used) were defined to achieve convergence of the computed electron densities during the time interval of the simulation. A few photoelectrons may leave the ionized cluster without being trapped by its Coulomb potential and will move tens to a few hundreds of Angstroms. Employing a fixed spatial step size of $0.08 \AA$ in agreement with the corresponding de

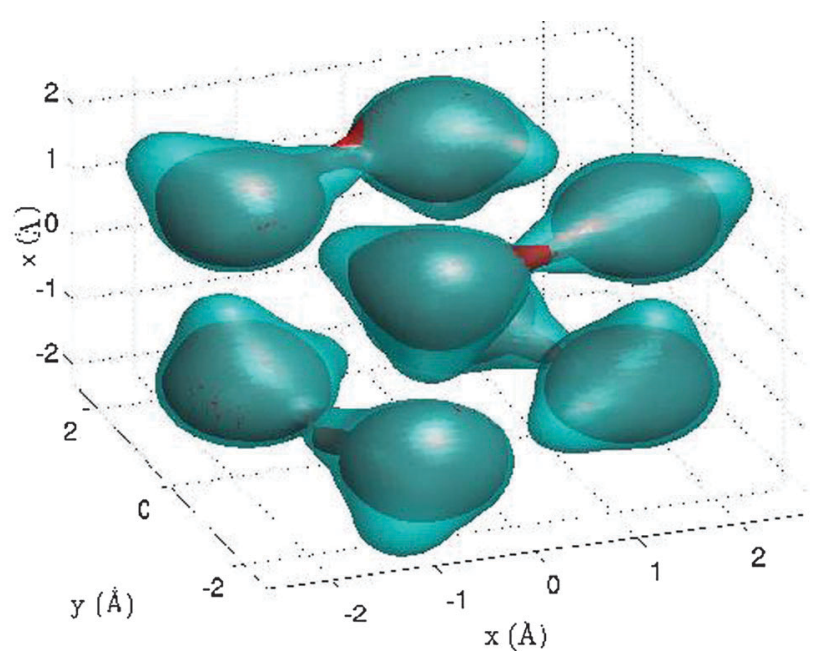

Fig. 2 Electron density change during the Coulomb explosion with a laser field intensity of $10^{16} \mathrm{~W} \mathrm{~cm}-2$. The isosurface value is for $\rho=0.6 \AA^{-3}$; the blue isosurface corresponds to the density at $0 \mathrm{fs}$, while the red one describes the density at $5 \mathrm{fs}$.
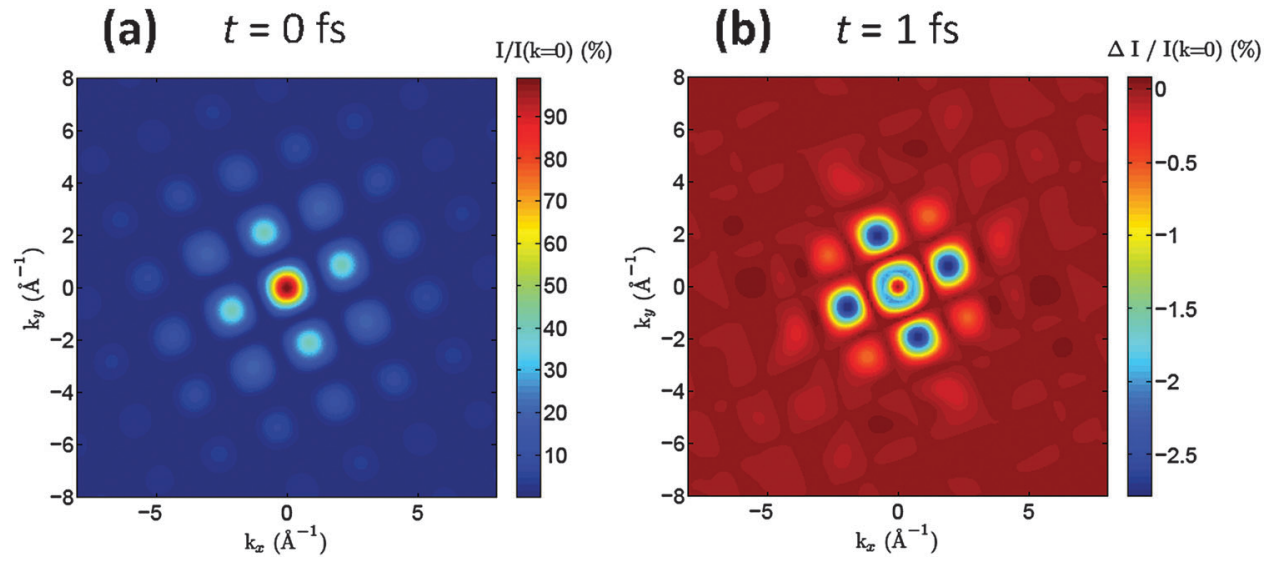

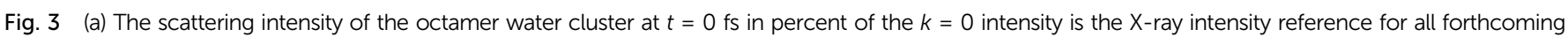

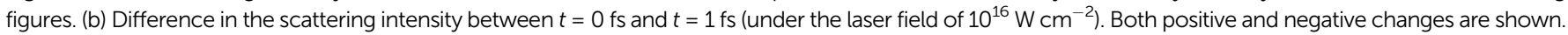


Broglie wavelength, we started with a box size of $10 \AA$ with the cluster center-of-mass located in the geometrical center of the box. Step by step, the size of the box was increased by $1.0 \AA$, and the convergence of the calculated densities was monitored up to $18 \AA$ A.

The simulations of the $\left(\mathrm{H}_{2} \mathrm{O}\right)_{8}$ water cluster in its electronic ground state start with the initial guess for the geometry optimization obtained from the Cambridge Cluster Database. ${ }^{21}$

\section{Results and discussion}

In X-ray structural measurements, the most important characteristic of a molecular system is the electron density and its possible time-dependent change. Specific features of electrongas formation in the sample during a Coulomb explosion can affect X-ray scattering to various degrees. From this point of view, an advantage of using TDDFT is that it offers $a b$ initio electron densities that follow the changes with time in detail.

Fig. 2 graphically displays changes in the electron density for the strongest field employed in our calculations $\left(10^{16} \mathrm{~W} \mathrm{~cm}^{-2}\right)$. The two isosurfaces shown correspond to an electron density of $0.6 \AA^{-3}$, with the blue isosurface belonging to the density at $t=0 \mathrm{fs}$, while the inner red isosurface gives the density at $t=5$ fs. It clearly can be seen that with the electron density becoming more diffuse the isovalue moves closer to the positions of the ions.

In order to evaluate the electron density changes in detail we have to switch from evaluation of the density in real space to Fourier space corresponding to scattering intensity spectra. A simple way to evaluate the significance of the structural damage caused by the laser field in structural measurements is the sensitivity of the X-ray scattering intensity to a change in the electron density, $\Delta \rho$. Due to the linearity of the Fourier transformation, the scattering intensity can be expressed simply by

$$
|F T(\rho(t)+\Delta \rho(t))|^{2}=|F T(\rho(t))+F T(\Delta \rho(t))|^{2} .
$$

We obtain the time-dependent valence electron (hydrogen electrons and $2 \mathrm{~s}$ and $2 \mathrm{p}$ electrons of the oxygen atoms) densities $\Delta \rho(t)$ from TDDFT calculations expressed on the spatial grid. Since these are smooth functions in comparison to the core electron densities, the Fourier transformation of these can be carried out conveniently using the fast Fourier transformation (FFT) method. The 1s core electron densities can be expressed using analytical functions, $\delta$-functions in the simplest approximation or correctly chosen $s$-functions. In the simplest approach, $F T(\rho(t))$ of the core electrons can be assigned to scattering factors and treated accordingly. This also separates the effects of the changes in valence electron density on the scattering spectra.
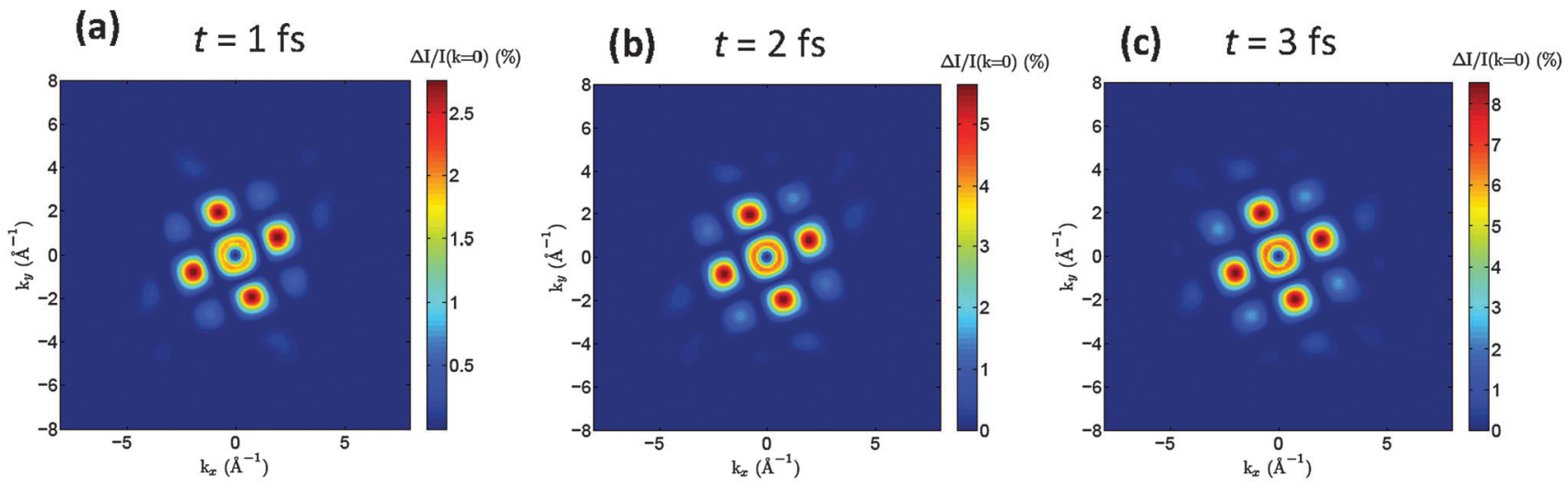

(d) $t=4 \mathrm{fs}$

(e) $t=5 \mathrm{fs}$
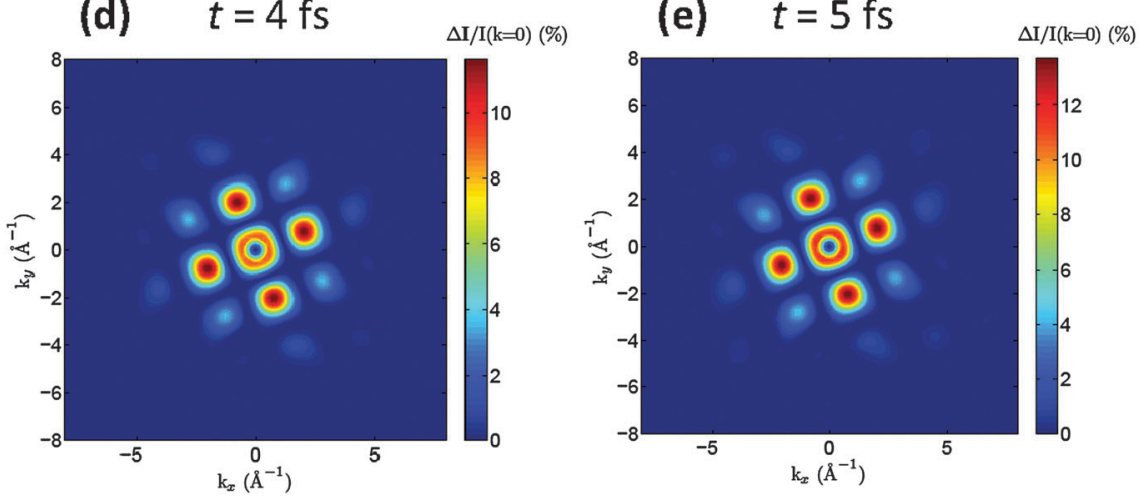

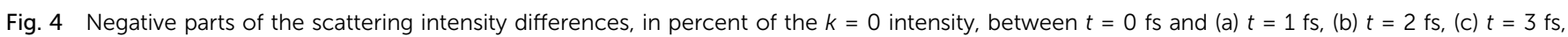
(d) $t=4 \mathrm{fs}$, (e) $t=5$ fs (under the laser field of $10^{16} \mathrm{~W} \mathrm{~cm}^{-2}$ ). 

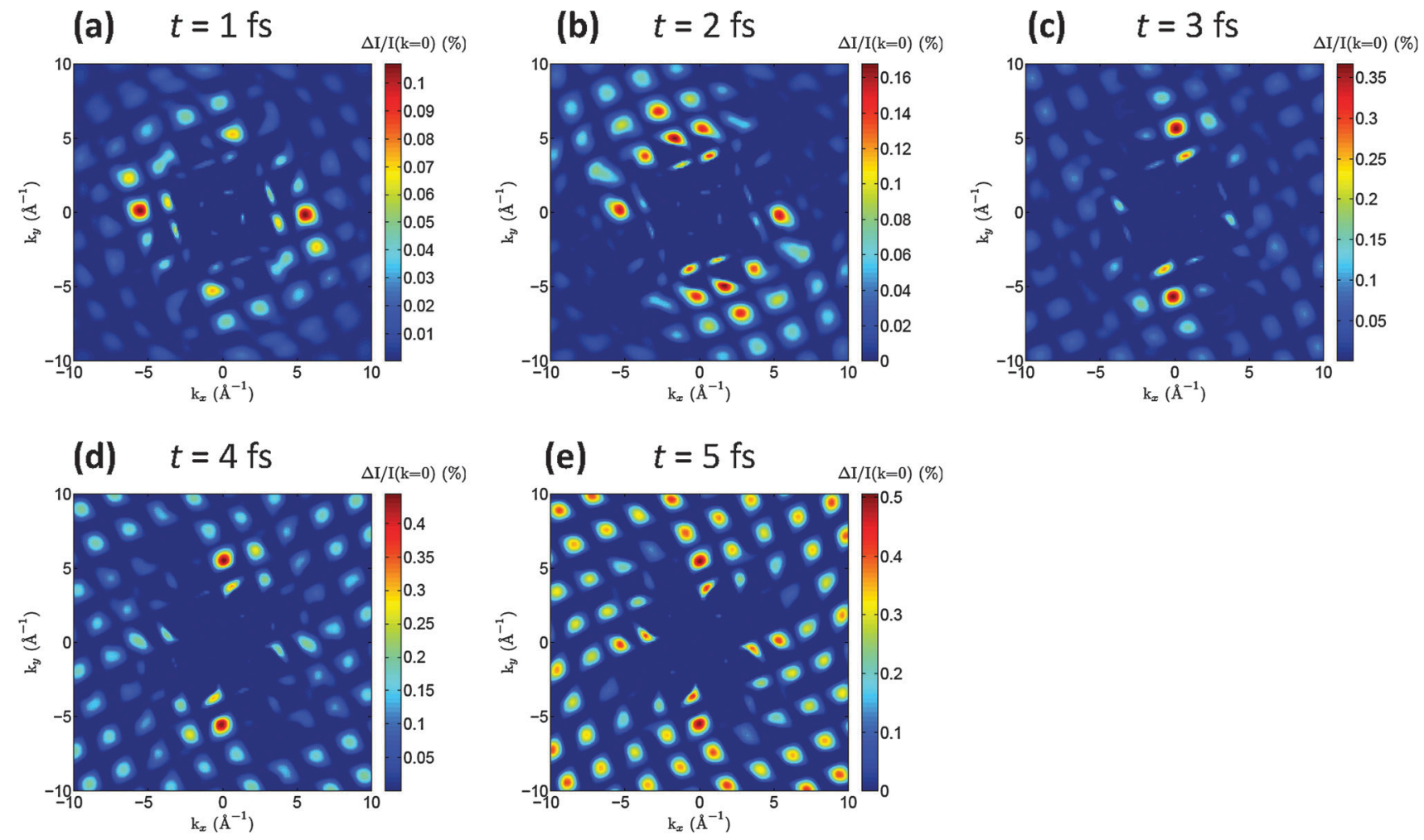

Fig. 5 Positive parts of the scattering intensity differences, in percent of the $k=0$ intensity, between $t=0 \mathrm{fs}$ and (a) $t=1 \mathrm{fs}$, (b) $t=2 \mathrm{fs}$, (c) $t=3 \mathrm{fs}$, (d) $t=4 \mathrm{fs}$, (e) $t=5 \mathrm{fs}$ (under the laser field of $10^{16} \mathrm{~W} \mathrm{~cm}^{-2}$ ).

Fig. 3(a) graphically displays the scattering intensity of the water cluster at $t=0$ as a reference for all further figures in this work. The intensity $I(k)$ is given in percent of $I(k=0)$, with the scattering vector $k=4 \pi \sin \theta \lambda^{-1}$, where $\theta$ denotes the scattering angle and $\lambda$ is the X-ray wavelength. The main maxima corresponding to the oxygen atoms are caused by the relatively higher number of electrons in comparison to the hydrogens. The hydrogen atoms which have no "core" electrons are included in the calculation in the electron density grid, an important fact to be kept in mind while examining the time-dependent scattering intensity evolvement. Fig. 3(b) shows the changes in the scattering intensity after $1 \mathrm{fs}$. There is a negative part, a depletion of the lower $k$-space, and an accumulation of intensity (positive part) in the region of higher $k$ values.

The time-dependent change of the intensity in the field of $10^{16} \mathrm{~W} \mathrm{~cm}^{-2}$ is depicted in Fig. 4 and 5 for $t=1, \ldots, 5 \mathrm{fs}$, separately for the negative (Fig. 4) and positive (Fig. 5) differences to $I(k=0, t=0)$. Noting the scales, it clearly can be seen that the four strong scattering maxima around $k=0$ are in a consistent fashion diminishing with time, while at the same time getting broader. The intensities from these maxima are redistributed to new features in the scattering map, especially in the higher $k$-range. After $t=5 \mathrm{fs}$, the scattering of these maxima decreases by about $13 \%$ of the $k=0$ scattering intensity. This means that in the strongest field the scattering intensity becomes gradually smeared out, being redistributed from the strong oxygen maxima close to $k=0$ to maxima at a higher $k$-range as the electrons start forming the quasi-free electron gas. When focussing on the part of the scattering spectra that corresponds to the forming electron-gas (Fig. 5), the scattering maxima first collect symmetrically around the main oxygen maxima. At later time-points, however, the electron-gas part of the scattering intensity spreads to a larger scattering vector range. The intensity changes shown for the field of $10^{16} \mathrm{~W} \mathrm{~cm}^{-2}$ are very strong, considering that the experimental X-ray probe pulse is a number of times longer than the 5 femtosecond time interval studied here. This shows that "inner ionized" electron gas is built up very fast, diminishing the scattering signal and degrading the scattering factors of the atoms in the sample.

The decrease of electron density in the inner part contributes to an instantaneous form factor decrease. The diffusion part finally contributes to an increase of mosaicity in the scattering patterns.

Finally, the negative and positive parts of the scattering intensity after 5 fs in the weaker field of $10^{15} \mathrm{~W} \mathrm{~cm}^{-2}$ are shown in Fig. 6. Although the atomic displacements ${ }^{3}$ in this field at this time-point are comparable to the displacements in the strongest field at about $2 \mathrm{fs}$, the scattering intensity is only half as influenced, dropping by only about $1.4 \%$ instead of $2.5 \%$. From this example the necessity for the knowledge of the exact electron density, and not just the change in atomic positions, becomes obvious. From the point of view of spectroscopic measurements, the weaker field shows a slightly more promising fragmentation rate. 
(a)

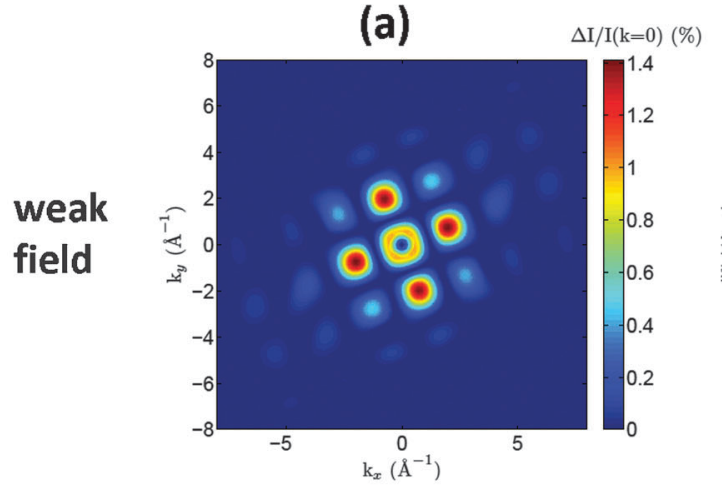

$\Delta \mathrm{I} / \mathrm{I}(\mathrm{k}=$

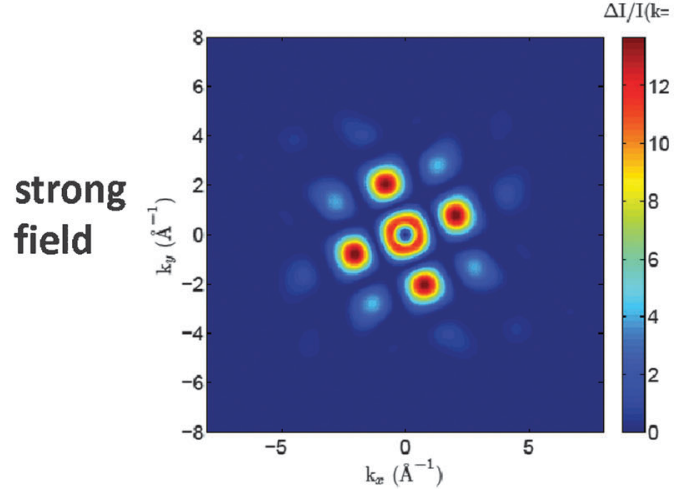

(b)
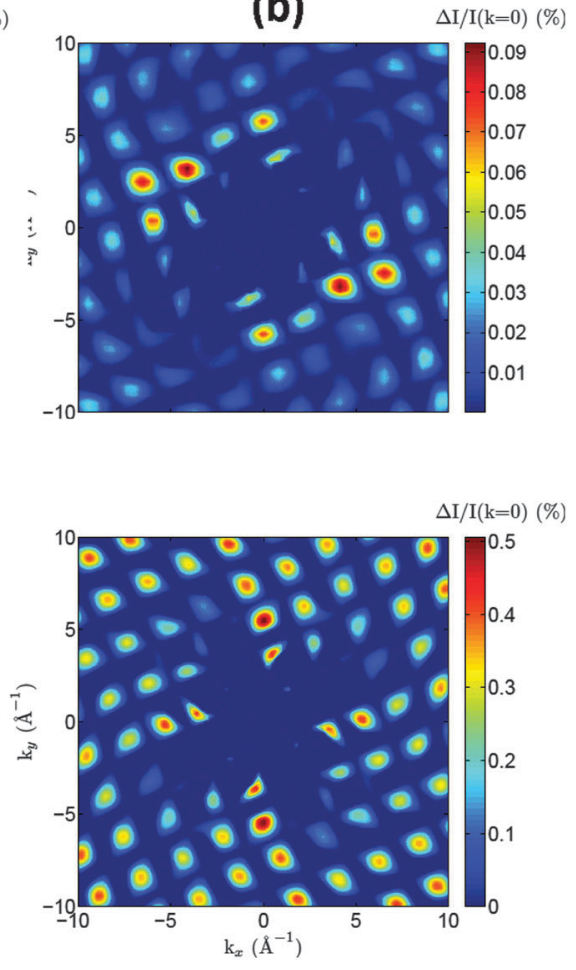

Fig. 6 Comparison of weak and strong fields: difference in scattering intensities (under the laser field of $10^{15} \mathrm{~W} \mathrm{~cm}^{-2}$ (top) and $\left.10^{16} \mathrm{~W} \mathrm{~cm}{ }^{-2}(\mathrm{bottom})\right)$ in percent of the $k=0$ intensity, between $t=0 \mathrm{fs}$ and $t=5 \mathrm{fs}$. (a) The negative part of the difference between the spectra and (b) the positive part of the difference between the spectra.

\section{Conclusions}

Theoretical investigations of the interaction between large molecular systems and X-ray lasers are still necessary. In the present work, we describe the X-ray laser-matter interaction in a quantum-mechanical ab initio approach, the size of the system under study has been limited to the small water cluster $\left(\mathrm{H}_{2} \mathrm{O}\right)_{8}$. Due to the dipole approximation and the description of atomic core electrons employing pseudopotentials, in the current implementation the OCTOPUS program package cannot take hard X-ray energies of the external laser pulse into account.

The overall symmetry and structure of the octamer water cluster during the Coulomb explosion is relatively well conserved on short time scales. ${ }^{3}$ The possibility to determine any structural details will be lost very fast due to the fast plasma formation. Although the actual ion movements during the first stages of the Coulomb explosion are minimal, especially for the heavier atoms ${ }^{3}$ the scattering intensity is very sensitive to the electron plasma build-up, as shown in Section III. Thus, it is important that simulations of Coulomb explosion accurately describe the detailed changes in the electron density.

As demonstrated in this work, one of the main advantages of TDDFT is the possibility to follow the $a b$ initio electron density of the system in detail as it changes in time under the influence of the laser field. The Coulomb explosion sets in with very fast electron plasma formation, the scattering signal of which contributes to the diffuse part of the scattering spectrum.
As has been shown, the scattering intensity on the main oxygen maxima drops by $12 \%$ in just 5 fs. The strongest field considered in the simulation $\left(10^{16} \mathrm{~W} \mathrm{~cm}^{-2}\right)$ leads to very fast cluster fragmentation, rendering structural measurements after a few femtoseconds useless.

A detailed ab initio analysis of the X-ray laser-matter interaction on small molecular systems can play an important role in choosing optimal field parameters for the structural measurements. TDDFT may become an important tool in estimating what field strengths and pulse lengths are necessary to get a scattering signal of needed quality. Time-dependent electrondensity measurements on the other hand can provide a further motivation for development of new density functionals.

The intensity in the region of smaller $k$ values changes mostly for times directly after the beginning of the Coulomb explosion. This smaller $k$-space corresponds to the electrondensity envelope of the molecular object, here the octamer water cluster. For longer times, in particular changes in the higher $k$-space are observed, while they are less pronounced in the lower $k$-space, i.e. in the envelope of the electron density. A negative change corresponds to a depletion of the molecular envelope.

\section{Acknowledgements}

This work was supported by DFG/SFB755 Nanoscale Photonic Imaging. S. T. thanks the Advanced Study Group of the Max 
Planck Society for continuous support. S. S. and S. T. are grateful for generous financial support by the Fonds der Chemischen Industrie.

\section{References}

1 U. Saalmann, C. Siedschlag and J. M. Rost, J. Phys. B: At., Mol. Opt. Phys., 2006, 39, R39.

2 K. Yamanouchi, Science, 2002, 295, 1659.

3 A. Debnarova, S. Techert and S. Schmatz, Phys. Chem. Chem. Phys., 2012, 14, 9606.

4 C. J. Gruenloh, J. R. Carney, C. A. Arrington, T. S. Zwier, S. Y. Fredericks and K. D. Jorda, Science, 1997, 276, 1678.

5 S. Karthikeyan, M. Park, I. Shin and K. S. Kim, J. Phys. Chem. A, 2008, 112, 10120.

6 Q. Du, E. Freysz and Y. Shen, Phys. Rev. Lett., 1993, 70, 2313.

7 B. Rowland, N. Kadagathur, J. Devlin, V. Buch, T. Feldman and M. Wojcik, J. Chem. Phys., 1995, 102, 8328.

8 G. Dixit and R. Santra, J. Chem. Phys., 2013, 138, 134311.

9 S. P. Hau-Riege, R. A. London and A. Soke, Phys. Rev. E, 2004, 69, 051906.

10 Z. Jurek, G. Faigel and M. Tegze, Eur. Phys. J. D, 2004, 29, 217.

11 Z. Jurek and G. Faigel, Europhys. Lett., 2004, 65, 491.

12 R. Neutze, R. Wouts, D. van der Spoel, E. Weckert and J. Hajdu, Nature, 2000, 406, 752.
13 M. Bergh, N. Timneanu and D. van der Spoel, Phys. Rev. E, 2004, 70, 051904.

14 A. Castro, H. Appel, M. Oliveira, A. Rozzi, X. Andrade, F. Lorenzen, M. A. L. Marques, E. K. U. Gross and A. Rubio, Phys. Status Solidi B, 2006, 243, 2465.

15 M. Marques, A. Castro, G. F. Bertsch and A. Rubio, Comput. Phys. Commun., 2003, 151, 60.

16 T. Ziegler, M. Seth, M. Krykunov and J. Autschbach, J. Chem. Phys., 2008, 129, 184114.

17 B. X. Yang and J. Kirz, Phys. Rev. B: Condens. Matter Mater. Phys., 1987, 36, 1361.

18 H. Hayashi, N. Watanabe, Y. Udagawa and C. Kao, Proc. Natl. Acad. Sci. U. S. A., 2000, 97, 6264.

19 N. Timneanu, C. Caleman, J. Hajdu and D. van der Spoel, Chem. Phys., 2004, 299, 277.

20 A. Thompson, D. Attwood, E. Gullikson, M. Howells, K.-J. Kim, J. Kirz, J. Kortright, I. Lindau, Y. Liu, P. Pianatta, A. Robinson, J. Scrofield, J. Underwood, G. Williams and H. Winick, X-ray data booklet, Lawrence Berkeley National Laboratory, University of California, 2009.

21 D. J. Wales, J. P. K. Doye, A. Dullweber, M. P. Hodges, F. Y. Naumkin, F. Calvo, J. Hernandez-Rojas and T. F. Middleton, The Cambridge Cluster Database, http://www-wales.ch.cam. ac.uk/CCD.html. 